\title{
Histoire du système éducatif sud-coréen
}

Tensions entre éducation égalitaire et différenciée

History of the South Korean education system. Recurring tensions between egalitarian and differentiated education

Historia del sistema educativo sur-coreano. Unas tensiones recurrentes entre educación igualitaria y diferenciada

\section{Chae-Chun Gim}

Traducteur : Sylvaine Herold

\section{(2) OpenEdition} Journals

Édition électronique

URL : https://journals.openedition.org/ries/6085

DOI : $10.4000 /$ ries.6085

ISSN : 2261-4265

Éditeur

France Education international

Édition imprimée

Date de publication : 1 décembre 2017

Pagination : 135-146

ISBN : 978-2-85420-616-6

ISSN : $1254-4590$

Référence électronique

Chae-Chun Gim, "Histoire du système éducatif sud-coréen », Revue internationale d'éducation de Sèvres [En ligne], 76 | décembre 2017, mis en ligne le 01 décembre 2019, consulté le 24 juin 2021. URL http://journals.openedition.org/ries/6085; DOI : https://doi.org/10.4000/ries.6085 


\title{
Histoire du système éducatif sud-coréen
}

Tensions entre éducation égalitaire et différenciée*

\author{
Chae-chun Gim \\ Université de Yeungnam, Corée du Sud
}

La Corée du Sud (Corée par la suite) a obtenu son indépendance du Japon en 1945 à la fin de la Seconde Guerre mondiale et a mis en place un système scolaire de type 6-3-3-4, composé de six années d'enseignement primaire, trois années de secondaire inférieur, trois années de secondaire supérieur et quatre années d'enseignement supérieur à l'université. À l'issue de la guerre de Corée, en 1953, l'enseignement primaire est devenu obligatoire et le taux de scolarisation au niveau du primaire a atteint $96 \%$ dès la fin des années 1950. L'enseignement secondaire inférieur s'est dès lors développé rapidement dans les années 1960 et 1970, pour devenir universel dans les années 1980, tandis que l'enseignement secondaire supérieur s'est accru rapidement dans les années 1970 et 1980 pour devenir universel au début des années 1990. L'enseignement secondaire inférieur est devenu obligatoire à partir de 1985 et a été mis en œuvre progressivement, en commençant par les établissements des zones reculées, jusqu'en 2004. Le plan national de 2017 prévoit par ailleurs que l'enseignement secondaire supérieur devienne obligatoire en 2020 et soit effectif d'ici 2022.

\section{LE CONTEXTE \\ DE LA FRAGMENTATION DE L'OFFRE ÉDUCATIVE EN CORÉE DU SUd}

On discerne aisément une tendance à la fragmentation de l'offre éducative dans les politiques éducatives coréennes. Celles-ci ont de tout temps oscillé entre une "éducation égalitaire " pour tous et une "éducation différenciée » correspondant aux capacités des élèves. Ce mouvement de balancier tire son origine de la Constitution même de la République de Corée. La Constitution, dans son article 31, section 1, stipule que "tous les citoyens doivent bénéficier d'un droit égal à recevoir une éducation correspondant à leurs capacités ${ }^{1}$. Ainsi empreintes de l'esprit de la Constitution, les politiques éducatives ont tour à tour oscillé entre le souci d'une " éducation égalitaire » pour tous et

\footnotetext{
* Article traduit par Sylvaine Herold.

1. La clause de la Constitution, dans sa version originale coréenne, est sujette à deux interprétations, le terme " égal » pouvant aussi bien s'appliquer à « droit » qu’à « éducation ».
} 
celui d'une "éducation différenciée " adaptée aux capacités des élèves. Avant la mise en œuvre de l'enseignement obligatoire au niveau du secondaire inférieur, les établissements d'enseignement souffraient du manque de financements publics, ce qui ne faisait qu'aggraver les disparités entre établissements riches et pauvres. Afin de combler cet écart, le gouvernement a mis en ouvre des politiques de standardisation de l'école, d'abord au niveau du secondaire inférieur en 1969, puis au niveau du secondaire supérieur en 1974. Ces politiques ont provoqué un profond changement dans les «systèmes d'exploitation » des établissements, qui sont passés d'une gestion "fondée sur le marché » à une gestion "fondée sur les politiques publiques». Les établissements, qui avaient jusqu'alors sélectionné leurs élèves et déterminé leurs frais de scolarité de manière autonome, n'ont plus été libres de le faire. Ils étaient désormais tenus d'accepter les élèves qui leur étaient attribués, ainsi que les frais de scolarité fixés par les bureaux d'éducation des districts. Cette volonté de standardisation de l'école montre que dans les années 1960 et 1970, les politiques éducatives coréennes ont accordé une plus grande importance à l'égalité en éducation qu'à une éducation "différenciée ».

Cependant, dans les années 1980, alors que les politiques de standardisation de l'école s'étaient ancrées en Corée, de nombreuses critiques se sont élevées à leur encontre, en particulier concernant le secondaire supérieur. Deux raisons principales étaient invoquées : tout d'abord, on reprochait à ces politiques de n'avoir pas permis de dispenser un enseignement adapté aux besoins et aux capacités des élèves; en second lieu, elles auraient conduit à une dégradation de la qualité de l'enseignement dans les établissements standardisés (Gim, 2017). En réponse à ces critiques, différents types d'établissements ont commencé à apparaître dans les années 1980 et 1990, tels que les « lycées ${ }^{2}$ spécialisés » (specialpurpose high schools), les lycées indépendants ou des écoles alternatives; puis, dans les années 1990 et 2000, les curricula différenciés et les « lycées à filière de spécialité »(subject-track high schools) ont également fait leur apparition. Cela illustre bien la manière dont les politiques éducatives coréennes ont changé de cap à partir des années 1980, passant d'un accent mis sur le «droit à l'égalité en éducation » au «droit à recevoir une éducation différenciée » correspondant aux capacités des élèves.

À l'heure actuelle, le nouveau Président de Corée du Sud, arrivé au pouvoir en mai 2017, semble être de tendance plus égalitaire que son prédécesseur et met de nouveau l'accent sur le droit à recevoir une "éducation égalitaire ». Dans ce contexte, le gouvernement actuel considère l' "éducation concurrentielle » comme un mal profondément ancré dans l'enseignement coréen et prévoit de supprimer les établissements " aristocratiques », tels que les lycées spécialisés en langue étrangère et les lycées indépendants, considérés comme la cause principale de la compétition excessive existant dans les établissements de

2. Nous emploierons le terme de «lycée " pour les établissements du secondaire supérieur. (NdT) 
l'enseignement obligatoire. Supprimer ou ne pas supprimer ces « établissements aristocratiques ", telle est aujourd'hui l'une des questions principales qui se pose au système éducatif coréen.

\section{UNE FRAGMENTATION DE L'OFFRE ÉDUCATIVE DE PLUSIEURS TYPES}

La grande majorité des écoles primaires de Corée sont des écoles publiques, seul un petit nombre d'entre elles (75 écoles, soit 1,2\% du total) ${ }^{3}$ sont des établissements privés ${ }^{4}$. Il a cependant existé en Corée un excédent relatif d'établissements privés au niveau du secondaire. En effet, l'enseignement secondaire s'est développé rapidement en Corée, et ce, avant même l'entrée en vigueur de l'enseignement obligatoire. Or, pendant la phase d'expansion de l'enseignement secondaire, entre les années 1960 et 1980, le gouvernement coréen ne disposait pas des fonds nécessaires pour bâtir des écoles. Il a donc mené une politique de "promotion de l'école privée ", en incitant les citoyens les plus riches à construire des écoles et en garantissant à ces écoles une aide financière du gouvernement. Ainsi, en 2017, il existe 640 établissements secondaires inférieurs privés, soit près de $20 \%$ de l'ensemble, et 949 établissements secondaires supérieurs privés, soit près de $40 \%$ de l'ensemble ${ }^{5}$.

Les politiques éducatives faisant la promotion d'une " éducation égalitaire » au niveau du primaire et du secondaire inférieur n'ont suscité que peu d'opposition, ces neuf années de scolarité faisant partie de l'éducation de base. La fragmentation de l'offre éducative a donc été limitée à ces niveaux. La situation diffère grandement au niveau du secondaire supérieur. Des décisions importantes sont prises en Corée pendant ces années d'enseignement, comme le choix d'une université ou d'un parcours professionnel. L'enseignement secondaire supérieur est dès lors devenu le théâtre de controverses brûlantes en lien avec la fragmentation de l'offre éducative. Dans cet article, nous examinerons principalement la fragmentation de l'offre éducative au niveau du secondaire supérieur.

En Corée, les politiques éducatives liées à la fragmentation de l'offre éducative sont de plusieurs ordres. En premier lieu, l'offre éducative s'est fragmentée sous l'effet de politiques de différenciation inter et intra-établissements. Certains dispositifs opèrent en effet une différenciation entre les établissements

3. Sauf indication contraire, l'ensemble des statistiques citées dans cet article proviennent du ministère de l'éducation et du KEDI (2016).

4. En Corée, les écoles primaires privées jouissent d'une grande autonomie, notamment pour sélectionner les élèves et fixer les frais de scolarité, autonomie dont ne disposent pas les établissements privés du secondaire depuis la politique de standardisation des années 1960 et 1970.

5. À mesure que l'urbanisation a entraîné le déclin du nombre d'élèves en milieu rural, le gouvernement a promu, dans les années 1990, une politique visant à faire d'un grand nombre d'établissements privés du secondaire des établissements publics, en reversant à leurs fondateurs un pourcentage des sommes qu'ils avaient initialement investies pour créer ces établissements. 
eux-mêmes, tandis que d'autres différencient les élèves au sein des établissements. Des écoles alternatives ont en outre été créées en Corée, par des ONG ou par de simples citoyens, pour promouvoir le développement de la personnalité 6 . En second lieu, une fragmentation plus traditionnelle opère selon le type de métiers auxquels se destinent les élèves : certains établissements sont destinés aux élèves envisageant de se rendre à l'université, tandis que d'autres forment ceux qui souhaitent travailler aussitôt après l'obtention de leur diplôme.

\section{FRAGMENTATION ENTRE ÉTABLISSEMENTS PRÉPARANT À L'ENSEIGNEMENT SUPÉRIEUR}

Des établissements d'enseignement promouvant le droit des élèves à choisir ont commencé à apparaître dans les années 1980 en Corée, comme alternatives aux établissements du secondaire supérieur standardisés introduits dans les années 1970. Les exemples les plus typiques sont: les lycées spécialisés, à partir des années 1980 ; les lycées indépendants privés, à partir des années 1990 ; et les écoles internationales, dans les années 2010 (Kim et Kim, 2015).

Avec la mise en ouvre de la standardisation des établissements du secondaire supérieur en 1974, l'ensemble des lycéens de Corée s'est mis à fréquenter des établissements de type homogène, à l'exception des élèves des lycées professionnels. Mais, à mesure que les critiques s'élevaient contre ces établissements, qui paraissaient peu adaptés aux besoins et compétences variés des élèves, le gouvernement a autorisé, à partir du milieu des années 1980, la création de "lycées spécialisés » ${ }^{7}$ comme les lycées scientifiques ou les lycées spécialisés en langues. Par la suite, les types de lycées spécialisés se sont diversifiés, pour inclure les arts et le sport au début des années 1990 et des lycées dits «internationaux » (global high schools) à la fin des années 1990.

Mais ces lycées spécialisés, en particulier en sciences et en langues, sont progressivement devenus des "établissements d'élite », réservés aux élèves cherchant à être admis dans des universités prestigieuses, notamment dans les facultés de médecine et de droit. Or, à mesure que la concurrence pour intégrer ces lycées d'élite s'intensifiait, l'éducation de l'ombre s'est également développée et des critiques ont souligné le fait que même les établissements du secondaire inférieur finissaient par devenir des écoles préparatoires pour les lycées d'élite. Il a été réclamé de manière de plus en plus pressante que les lycées spécialisés reviennent à leur mission initiale, des établissements dans lesquels les élèves aux intérêts et aux talents particuliers dans des matières données sont admis afin de pouvoir étudier de manière approfondie ces sujets. Le gouvernement a alors adopté deux décrets limitant, depuis les années 2000, d'une part, le nombre de candidats issus

6. Character development dans le texte $(N d T)$.

7. Special-purpose high schools dans le texte (NdT). 
des lycées scientifiques dans les facultés de médecine et, d'autre part, le nombre de candidats issus des lycées spécialisés en langues en dehors des départements de langues et d'humanités. En 2017, il existe 28 lycées scientifiques (1,2\% des établissements du secondaire supérieur) et 31 lycées spécialisés en langues (1,3\%).

Par ailleurs, avec l'entrée en vigueur de la politique de standardisation des établissements du secondaire supérieur en 1974, les établissements privés ont perdu leur autonomie en matière de sélection des élèves et de fixation des frais de scolarité. Les établissements privés ont dès lors dû accepter les élèves qui leur étaient attribués par les bureaux municipaux/provinciaux de l'éducation et ils ont également dû appliquer les mêmes frais de scolarité que les établissements publics. En contrepartie, le gouvernement a mis en place une politique de soutien financier à destination des établissements privés $^{8}$, qui a abouti à ce qu'aucune différence substantielle n'existe à ce jour entre les lycées privés et les lycées publics. Néanmoins, afin de faire face au mécontentement grandissant des établissements privés, qui avaient été privés de leur autonomie, le gouvernement a adopté, au début des années 2000, une politique autorisant la création de «lycées indépendants ${ }^{9}$ (Gahng, 2015). Par cette mesure, les lycées privés pouvaient regagner le droit de sélectionner leurs élèves et de fixer leurs propres frais de scolarité s'ils renonçaient au soutien financier accordé par le gouvernement. Il existait jusqu'en 2009 six lycées indépendants, opérant à l'échelle nationale. Puis, à partir de 2009, les lycées indépendants se sont développés rapidement à la condition de recruter leurs élèves dans les limites de leurs régions. Les six premiers lycées indépendants, autorisés à recruter leurs élèves à l'échelle nationale, sont devenus des établissements d'élite et ont été critiqués pour être "aristocratiques ", les frais de scolarité pratiqués étant trois fois supérieurs à ceux d'un établissement public, sans compter les frais d'internat élevés. En 2017, il existe 46 lycées indépendants en Corée (soit $2 \%$ des établissements du secondaire supérieur).

Au début des années 2010, le gouvernement coréen a mis en œuvre une politique de promotion des «écoles internationales », faisant de l'île de Jeju (l'une des neuf provinces de Corée) « la ville de l'enseignement en anglais ${ }^{10}$ » et invitant de prestigieux établissements secondaires supérieurs des pays développés à venir y établir des succursales. Cette politique était destinée, d'une part, à stimuler les établissements coréens en attirant des établissements prestigieux; d'autre part, à inciter les élèves coréens partis étudier à l'étranger à revenir en Corée. Plusieurs établissements internationaux sont aujourd'hui présents sur l'île de Jeju ; par exemple : la North London Collegiate School de Grande-Bretagne, le Branksome Hall du Canada et la Saint Johnsbury Academy des États-Unis.

8. Il est dans les faits impossible de faire fonctionner un établissement privé sur la base des mêmes frais de scolarité qu'un établissement public ; le ministère de l'éducation offre donc une aide financière aux établissements privés, qui comprend les salaires des enseignants et les coûts d'exploitation.

9. Independent high schools, dans le texte (NdT).

10. "Jeju English Education City » dans le texte. (NdT) 
Les établissements internationaux présents à Jeju diffèrent des autres écoles internationales présentes en Corée, notamment parce qu'ils ont le droit de sélectionner jusqu'à $100 \%$ d'élèves coréens dans leurs quotas d'entrées et qu'ils peuvent délivrer des certifications approuvées par le gouvernement, à condition que les élèves aient suivi les cours de langue et d'histoire coréennes. Ainsi, les établissements internationaux de Jeju accueillent une majorité d'élèves coréens, et non des élèves internationaux. Ils mettent en œuvre un enseignement de type K-12 11 et leurs diplômés poursuivent en général leurs études dans des universités internationales plutôt que dans les universités nationales. La présence de ces établissements n'a cependant pas eu l'impact escompté sur la société coréenne en raison de leur localisation, de leurs frais de scolarité élevés et du choix fait par leurs diplômés de fréquenter des universités étrangères.

\section{FRAGMENTATION ENTRE ÉTABLISSEMENTS PRÉPARANT À L'EMPLOI}

Dans les années 1960, la Corée a adopté un système scolaire dual composé de lycées généralistes et de lycées professionnels ${ }^{12}$. Jusque dans les années 1980, les élèves étaient libres de choisir entre un enseignement général en humanités dans des lycées généralistes et une formation à un métier dans des lycées professionnels (Lee, 2017). Au cours des années 1960 et 1970, les bons élèves étaient tout aussi susceptibles de choisir un lycée professionnel qu'un lycée généraliste. Et dans les faits, ces élèves, qui avaient choisi volontairement de poursuivre leur scolarité dans des lycées professionnels au cours des années 1960 et 1970, ont amplement contribué au développement actuel de l'économie coréenne, centrée sur l'industrie manufacturière. Mais, à mesure que se développait l'enseignement universitaire dans les années 1980, cette relation équilibrée s'est transformée en une relation hiérarchique, dans laquelle les meilleurs élèves choisissent les lycées généralistes pour se préparer à l'université, tandis que les élèves moins aptes vont dans des lycées professionnels pour se former à un métier.

En 2005, le gouvernement coréen a cherché à diversifier l'enseignement secondaire professionnel en initiant un processus de transformation des lycées professionnels traditionnels/généraux, qui formaient une main-d'œuvre aux compétences de base, en des lycées professionnels spécialisés, formant leurs élèves afin qu'ils deviennent des experts dans des secteurs spécifiques, comme l'automobile, la robotique, la conception, la musique pop et l'animation. Ainsi, la formation professionnelle au niveau du secondaire supérieur est elle aussi devenue bipartite avec, d'un côté, les lycées professionnels généraux et, de l'autre, les lycées professionnels spécialisés. En 2010, tous les lycées professionnels

11. L'acronyme « K-12 » désigne en anglais les niveaux scolaires de la maternelle (Kindergarten) jusqu'à la $12^{\mathrm{e}}$ année d'enseignement, correspondant à la fin du secondaire supérieur. $(N d t)$

12. Il n'existait qu'un seul type d'établissement secondaire supérieur en Corée depuis l'indépendance en 1945. 
traditionnels avaient évolué en des lycées professionnels spécialisés (Kim et Kim, 2015). À la même époque, le gouvernement coréen a décidé d'instaurer un nouveau type d'établissements secondaires supérieurs professionnels, dénommés les «lycées Meister » ${ }^{13}$ dans le but d'atténuer les réticences des meilleurs élèves envers l'enseignement professionnel et de favoriser la formation de techniciens professionnels afin d'établir les fondements de diverses industries coréennes. Des lycées Meister ont été créés dans plusieurs secteurs industriels : la mécanique, l'électronique, les bioindustries, l'industrie de l'acier et la pétrochimie. Ces lycées sont parvenus à attirer les bons élèves grâce à un certain nombre de mesures : en dérégulant les qualifications des enseignants et des directeurs d'établissements $^{14}$; en améliorant les équipements et les locaux ; en renforçant la formation sur le lieu de travail ; et en réduisant le nombre d'élèves par classe. En 2017, il existe 472 lycées professionnels spécialisés (soit $20 \%$ de l'ensemble des établissements du secondaire supérieur) et 42 lycées « Meister» $(1,8 \%)$.

\section{DÉVELOPPEMENT D'ÉCOLES « HORS SYSTÈME »}

Au début des années 1990, le nombre croissant d'élèves qui abandonnaient l'école voire se suicidaient ${ }^{15}$ en raison de leur incapacité à s'adapter au style d'enseignement implacablement uniforme et oppressif pratiqué dans les établissements standardisés est devenu un enjeu social majeur en Corée. Des écoles alternatives sont alors apparues pour accueillir les élèves non adaptés à ce système. Ces écoles alternatives, créées par des ONG ou par de simples citoyens, agissent en dehors du contrôle gouvernemental et ne sont donc pas autorisées à

13. Meister high schools dans le texte. Ce réseau d'établissements, créé en 2010, vise à préparer les jeunes à des emplois hautement qualifiés dans le domaine manufacturier ou d'autres. Il vise également à améliorer la perception du statut de ces positions. Source : Mc Kisey, voir : goo.gl/ZxqsWA (NdIR)

14. Cette dérégulation a permis à des professionnels issus des secteurs industriels concernés et ne disposant pas des certificats d'enseignement de devenir chefs d'établissement et/ou enseignants dans les lycées Meister.

15. Le tableau suivant donne les taux d'abandon scolaire et de suicide pour les élèves du secondaire inférieur et du secondaire supérieur.

\begin{tabular}{|c|c|c|c|c|c|c|c|c|c|c|c|c|c|c|c|c|}
\hline \multicolumn{2}{|c|}{ Année } & $\stackrel{1}{\circ}$ & $\begin{array}{l}\infty \\
\stackrel{\infty}{2}\end{array}$ & ஓి & ஓ & $\bar{\sigma}$ & ๙ૂ & ๙̊ & Бૅ & $\stackrel{n}{\Omega}$ & よ̊ & $\hat{\sigma}$ & ๙ & $\vdots$ & nn & ำ \\
\hline \multirow{2}{*}{$\begin{array}{l}\text { Taux } \\
\text { d'abandon } \\
(\text { en \%) }\end{array}$} & $\begin{array}{l}\text { Secondaire } \\
\text { inf. }\end{array}$ & 1,2 & 1,1 & 1 & 1 & 0,9 & 0.9 & 0,7 & 0.8 & 1 & 1,1 & 1.2 & 1 & & 0,6 & 0,6 \\
\hline & $\begin{array}{l}\text { Secondaire } \\
\text { sup. }\end{array}$ & 2,6 & 2,4 & 2,2 & 2,4 & 2,2 & 2,2 & 2,1 & 2,2 & 2,5 & 2,6 & 2,7 & 2,1 & & 1,3 & 1,4 \\
\hline \multirow{2}{*}{$\begin{array}{l}\text { Taux } \\
\text { de suicide } \\
\text { (pour } \\
100000 \text { ) }\end{array}$} & $\begin{array}{l}\text { Secondaire } \\
\text { inf. }\end{array}$ & 1 & 0,7 & 0,9 & 1,1 & 1,1 & 1,2 & 1 & 1,3 & 1,5 & 2,2 & 1,8 & 2,1 & & 1,2 & 0.9 \\
\hline & $\begin{array}{l}\text { Secondaire } \\
\text { sup. }\end{array}$ & 5,6 & 6,7 & 6 & 6,3 & 5,6 & 7,2 & 7,5 & 6,6 & 7,5 & 10,4 & 7,6 & 9,6 & & 6,5 & 7,9 \\
\hline
\end{tabular}


délivrer les certifications officielles. Elles favorisent un enseignement respectueux de l'environnement et collaboratif, plutôt qu'une formation académique standard. Leur nombre et leur type ont rapidement augmenté et il existe par exemple de nos jours : des écoles alternatives pour la formation professionnelle dans l'agriculture et la foresterie; des écoles qui promeuvent une éducation tournée vers l'épanouissement personnel; des écoles favorisant certaines perspectives religieuses; et des écoles imitant les écoles internationales.

À la fin des années 1990, le gouvernement a introduit un dispositif d' " accréditation des écoles alternatives », par lequel les écoles alternatives accréditées par le gouvernement peuvent recevoir une aide financière si elles répondent aux normes fixées (Lee, Hwang, Kang et Ha, 2009). Mais, même ainsi, de nombreuses écoles alternatives choisissent de demeurer non accréditées, afin de pouvoir gérer leur établissement en dehors de toute contrainte gouvernementale. Les élèves fréquentant ces écoles alternatives non accréditées doivent passer un examen, administré par le gouvernement, afin de valider leurs certifications. Mais cet examen n'est pas excessivement difficile et les élèves des écoles alternatives non accréditées obtiennent aisément les certifications officielles.

\section{FRAGMENTATION INTERNE AUX ÉTABLISSEMENTS PRÉPARANT À L'ENSEIGNEMENT SUPÉRIEUR}

L'enseignement secondaire supérieur a longtemps été non diversifié en Corée : les mêmes contenus d'enseignement étaient dispensés au même rythme et selon les mêmes méthodes à une classe d'élèves hétérogènes. Cela conduisait rapidement les bons élèves à s'ennuyer tandis que les élèves ayant des difficultés d'apprentissage peinaient à suivre. Pour remédier à cette situation, le gouvernement a décidé, à partir de la fin des années 1990, de mettre en ouvre des curricula différenciés au sein des établissements. Les élèves ont dès lors été répartis en cours de niveau, en maths ou en anglais notamment, matières dans lesquelles les résultats des élèves étaient les plus disparates. Mais ce nouveau dispositif s'est rapidement confronté à la problématique de l'évaluation, car les élèves répartis en classes de niveau - comprenant généralement des classes avancées, intermédiaires et de rattrapage - étaient néanmoins évalués selon les mêmes examens (Gim, 2000), le classement relatif des élèves ou leur note relative dans chaque matière étant nécessaire pour postuler et être admis à l'université. Ainsi, tous les élèves étaient évalués selon un examen identique, quelle que soit la classe de niveau à laquelle ils avaient été affectés.

Cette situation a de surcroît engendré une grande confusion chez les élèves, qui devaient se rendre dans des salles de classe différentes pour les cours de maths et d'anglais. Le gouvernement a alors introduit, en 2008, un système de "salle de classe par matière ", qui est venu remplacer une longue tradition de «salle de classe principale » en Corée. Traditionnellement, en Corée, les élèves 
se voyaient affecter une salle de classe principale et les enseignants se déplaçaient d'une salle à l'autre pour donner leurs cours. Dans un système de " salle de classe par matière ", au contraire, des salles de classe sont attribuées à chaque matière et les élèves se déplacent d'une salle à l'autre pour suivre leurs cours. En bref, les enseignants se déplacent d'une salle à l'autre dans un système de salle de classe principale, tandis que ce sont les élèves qui se déplacent dans un système de salle de classe par matière. Or les élèves, qui s'étaient habitués au système traditionnel, ont trouvé déroutant de devoir se déplacer d'une salle à l'autre, notamment au cours de la période transitoire entre la mise en ouvre des curricula différenciés et l'entrée en vigueur du système de salle par matière. En outre, un grand nombre d'établissements du secondaire supérieur ne sont parvenus à mettre en œuvre ce système que pour les cours de niveau en maths et en anglais, en raison du manque de salles de classe disponibles.

En 2009, le gouvernement a mis en œuvre un dispositif de «filières de spécialité » dans les établissements du secondaire supérieur, permettant aux lycées généralistes d'ouvrir des filières d'enseignement spécifiques pour un enseignement intensif dans certaines matières (Han, 2012). En effet, face au nombre croissant d'élèves désireux de se rendre dans des lycées spécialisés, le gouvernement a dû choisir entre accroître le nombre de lycées spécialisés ou instaurer des filières similaires au sein des lycées généralistes. Le ministère de l'éducation a finalement penché pour la seconde option car augmenter le nombre de lycées spécialisés n'aurait fait qu'aggraver la concurrence déjà excessive existant dans l'enseignement secondaire, tout en accablant toujours plus les parents et les élèves du fardeau du tutorat privé.

Un "lycée à filière de spécialité " ${ }^{16}$ est un lycée généraliste qui propose, en plus, une filière d'enseignement de spécialité dans une discipline spécifique, dont le contenu est semblable aux curricula proposés par les lycées spécialisés de ces mêmes domaines. Ces filières fonctionnent donc un peu comme une école au sein de l'école. Ainsi, un lycée à filière de spécialité en sciences propose un double curriculum : un curriculum à contenu intensif en sciences pour les élèves de la filière de spécialité et un curriculum normal/standardisé pour les autres. Ainsi, bien qu'ils fréquentent un lycée généraliste, les élèves de la filière de spécialité suivent un programme similaire à celui d'un lycée spécialisé en sciences.

En 2017, il existe 135 lycées proposant une filière de spécialité en sciences, 75 en anglais, 20 en sciences sociales, 9 en seconde langue et 19 en sports et en arts. Ces lycées à filière de spécialité font figure de formidable réussite car ils permettent aux élèves n'ayant pas la possibilité d'intégrer un lycée spécialisé, en raison de quotas d'entrée restreints, de suivre une filière de spécialité dans un lycée standard et, par là même, de satisfaire leur désir d'apprendre grâce à des programmes avancés dans leurs disciplines d'intérêt.

16. Subject-track high school dans le texte $(N d T)$. 


\section{REMARQUES FINALES}

Depuis 70 ans qu'existe le gouvernement de Corée du Sud, les politiques éducatives coréennes ont oscillé entre une "éducation différenciée » et une "éducation égalitaire ». De nos jours, le pendule semble s'éloigner de l' « éducation différenciée adaptées aux capacités » pour tendre de nouveau vers l'«éducation égalitaire pour tous ». Ces oscillations répétées ne signifient pourtant pas que l'on rejoue sans fin les mêmes politiques éducatives. En effet, quel que soit le pôle vers lequel on tend, le système éducatif coréen s'est développé progressivement dans le sens d'une éducation personnalisée. Avec le développement des technologies de l'information et de la communication (TIC) et l'émergence de l'intelligence artificielle, l'éducation personnalisée est de surcroît devenue un objectif plus atteignable que jamais.

Récemment, l'actuel président de Corée a présenté la mise en œuvre d'un "système de crédits pour le secondaire supérieur " comme sa priorité en matière d'éducation. Ce dispositif offre aux élèves la possibilité de choisir leurs cours parmi différents niveaux d'études et différentes filières d'enseignement, leur permettant ainsi d'opter pour les matières qui les intéressent le plus afin d'atteindre le nombre de crédits nécessaires à l'obtention du diplôme. Mais pour que ce système réussisse, deux conditions au moins paraissent nécessaires : les établissements doivent être en mesure de proposer plusieurs options intéressant les élèves, mais également d'organiser un système de salles de classe par matières pour permettre aux élèves de suivre leurs cours. Or, pour parvenir à proposer un éventail de choix plus vaste aux élèves coréens, plusieurs mesures doivent être prises. Voici quelques-unes des options actuellement à l'étude : la mise en œuvre de programmes d'études conjoints entre établissements ; l'enseignement en ligne des cours optionnels; la création d'un centre d'apprentissage commun en dehors de l'établissement. Pour faciliter le bon fonctionnement de ce système de crédits, on devrait également, avant toute chose, mettre un terme aux pratiques d'évaluation qui consistent à classer les élèves en fonction de leur rang ou de leurs notes.

Finalement, comment choisir en Corée entre une "éducation égalitaire » et une "éducation différenciée" ? Quelle est l'option la plus souhaitable: regrouper les élèves ayant des besoins, des intérêts et des aptitudes similaires dans des classes distinctes et leur dispenser un enseignement différencié, ou bien réunir des élèves aux besoins, intérêts et aptitudes pluriels sous un même toit et leur dispenser le même enseignement ? S'il existe une troisième voie, quelle est-elle ?

L’actuel président de Corée s'est engagé, pendant la campagne électorale, en faveur d'une éducation égalitaire. C'est dès lors devenu une priorité du gouvernement actuel, qui envisage très sérieusement de supprimer les lycées spécialisés et les lycées indépendants, qui sont considérés comme la cause principale de la concurrence démesurée existant au niveau du secondaire et du recours excessif au tutorat privé. Dans les cinq années à venir, le système éducatif coréen devrait donc évoluer dans le sens d'une éducation plus égalitaire. 


\section{BIBLIOGRAPHIE}

GAHNG Tae-Joong (2015) : "A review of the discourses in South Korea on the high school system »(en coréen), Educational Research and Practice, n ${ }^{\circ}$ 81, p. 1-26.

GIM Chae-Chun (2017) : "A study on the change of educational policies in preprimary, primary and secondary schools» (en coréen), in KIM Shin-Bok (eds.), Changes and prospect of educational policies, Séoul : Seoul National University Press, p. 77-155.

GIM Chae-Chun (2000): Differentiated curriculum (en coréen), Séoul : Gyoyookkwahaksa.

HAN Hye-Chong (2012) : "A study on the curriculum organization and implementation of the subject-specialized high school » (en coréen), The Journal of Curriculum Studies, $\mathrm{n}^{\circ}$ 30(1), p. 99-124.

KIM Seong-Yul et KIM Hoon-Ho (2015) : «Diversification of high school system in Korea - Changes, performances and challenges"(en coréen), Educational Research and Practice, $\mathrm{n}^{\circ}$ 81, p. 27-56.

LEE Hye-Young, HWANG Joon-Sung, KANG Dae-Joong et HA Tae-Wook (2009) : "A study on the current state analysis of alternative schools" (en coréen), KEDI Research Report (RR 2009-03), Séoul : Korean Educational Development Institute.

LEE Moo-Geun (2017) : "Changes and prospect of vocational education policies " (en coréen), in KIM Shin-Bok (eds.), Changes and prospect of educational policies, Séoul : Seoul National University Press, p. 275-347.

MOE (Ministry of Education) \& KEDI (Korean Educational Development Institute) (2016) : Statistical Yearbook of Education.

STATISTICS KOREA (2016) : Korean statistical information service. 
